\title{
« Le souvenir n'est qu'une maison que l'on porte dans la voix » (Alexandre Amprimoz, Bouquet de signes)
}

Sante A. VISELLI, Université de Winnipeg

Dans cette étude, je souhaite rendre hommage à notre collègue et ami Alexandre Laurent Amprimoz et le remercier à ma manière de son soutien et de son encouragement au cours de ma carrière, mais surtout de son amitié inconditionnée.

Depuis déjà plusieurs années, nous remarquons au Canada la présence d'une littérature unique en son genre, celle des auteurs italo-canadiens. En français, en anglais ou en italien, cette littérature s'est déjà frayé un chemin sûr vers une reconnaissance incontestée au niveau national aussi bien qu'international. Des auteurs tels que Fulvio Caccia, Antonio d'Alfonso, Marco Micone, Carole David-Fioramore, Bianca Zagolin et Alexandre Amprimoz, sont à considérer parmi les lauréats italo-canadiens, porte-parole d'une génération d'érudits cosmopolites et polyglottes. L'œuvre d'Amprimoz est, à cet égard, un exemplum des plus marquants d'une littérature multiple et plurielle (Viselli 24-27).

Je partage avec mon ami mes origines. Le Latium fut notre berceau commun. Né à Rome de père français et de mère italienne, Alexandre L. Amprimoz porte en lui les signes d'une double appartenance, d'une double sensibilité. Sans cette clef, il est impossible d'apprécier sa « voix », son désir inassouvi d'écrire : l'écriture devient la traduction du drame de celui qui cherche à sortir de son état, de ses peurs, de ses rêves, du souvenir qui le hante et qu'il féconde. Cependant, contrairement à tant d'auteurs franco-italo-canadiens, il lance dans son œuvre surtout poétique un défi au mutisme et à la mort, je dis mutisme et pas silence, car en bon symboliste Amprimoz savait bien jouer sur les nuances sémantiques relatives aux deux signes. Le souvenir sera toujours présent sous sa plume souvent tourmentée :

le long des rues parmi les parfums de l'ail

et des pierres assassinées de lumière se perd le goût aigre du temps

le passé est un musée trop vieux et trop compliqué pour comprendre le ciel sans nuages qui poignarde de son bleu le présent ah oui le ciel romain où Dieu sourit («VI », Chant solaire, 29) 
Or, le poète subira des avatars nécessaires à sa création, à sa quête, à son salut : c'est en philosophe et en véritable poète qu'il réussira à sortir de l'impasse de son labyrinthe intérieur et à dépasser le jeu quelque peu romantique, le mal du pays de tant d'émigrés. Sans être une prison, le souvenir deviendra l'isotopie dominante pour atteindre, dans sa poésie, une réalité autre, « une maison » construite par sa «voix» («L'autre maison», Bouquet, 90). Le poète, tel un Victor Hugo, a une tâche à accomplir même si cela implique un changement de direction, car, comme il me le répétait souvent dans nos conversations : «Il n’y a que les ânes qui ne changent pas d'avis ». Devant l'éphémère qui hante l'être et le bafoue, la souffrance trace la méthode de la recherche à poursuivre :

Traverser la rue. Notre monde serait-il l'illusion d'une éternelle éclipse ?

Tâche d'être touché par la souffrance de cet arbre et tu comprendras l'univers. C'est ici qu'un Dieu lointain te fait signe.

Poète, marche sur les mots comme sur la glace à l'aube du printemps.

Tu as enfin trouvé le privilège : le changement de ton. («7 7 , Changements de tons, 83).

Le poète s'enivre dans l'évocation surréelle d'images juxtaposées (la neige, les tournesols) et dans la certitude, j'ose dire cartésienne, d'une réalité dont il serait « l'artisan », le créateur ultime. «C'est ici qu'un Dieu / lointain te fait signe », dit-il dans le poème cité. Cet « ici »n'est plus l'ailleurs qui le tourmente et le confond : « ici » est où le poète taille ses formes et façonne ses vers, avec ménagement, car la glace sur laquelle il marche est devenue fragile et instable. Malgré cette fragilité, le printemps amprimozien annonce cependant la lumière, la promesse d'une poésie renouvelée.

Tout s'estompe ainsi, grâce à la certitude d'une autre existence qui sera peut-être un jour la tienne.

Être, même si ce n'est plus tellement à la mode. Pouvoir vivre ses impressions. Dire comment la neige caresse les futurs champs de tournesols. Être l'artisan d'un texte. Respirer la satisfaction de l'œuvre finie.

Laisser la nuit devenir ton texte. Connaître le savoir du travail. Déjà tu vois venir un autre monde. $(\ll 8 », 84)$.

«Déjà tu vois venir un autre monde » : si le poète croit qu’à la base de toute création poétique il y a l'angoisse, contrairement à un Apollinaire, 1'«Espérance » en est, pour Alessandro, la 
compagne et elle n'est pas violente. Héritier du pays du soleil, il en est en même temps le symbole et l'antithèse. Il porte en lui l'expérience de la mort et la joie de vivre des méridionaux, un phénomène culturel d'ailleurs bien connu en Italie. Dans ces villages à l'air amorphe et désertés par ses enfants à la recherche d'une vie meilleure ailleurs, c'était la coutume de traiter le départ des émigrés comme une mort symbolique dans la communauté, car, comme le dit le proverbe : partir, c'est toujours mourir un peu. Alexandre devait bien connaître cet usage, lui si sensible à cet aspect dramatique de la condition humaine (Viselli 28). Avant de rentrer en France avec sa famille, après son séjour à Palavas-les-Flots près de Montpellier, puis à Paris et à Aix-enProvence, il avait vécu à Rome, sa ville natale, et en Toscane. Encore jeune et comparable à pas mal d'entre nous de cette génération, il ne sera pas sourd à l'appel d'une liberté rêvée, à l'invitation de son «bateau ivre » qui le séparera définitivement un jour de l'Italie et de la France. C'est l'appel de l'inconnu grâce auquel l'enfant et l'exilé, - le futur professeur d'université, cet émigré qui n'émigre certainement pas pour des raisons économiques -, se rejoignent dans la même blessure, celle causée par le mal du pays et par la prise de conscience que le retour ne pourra ni ne devra se concrétiser :

c'est le navire

que l'enfant salue au bord du caniveau avant que la coque d'un vieux devoir ne s'éprenne du pays des ordures

et la splendeur d'un ancien théorème ira importuner les rats

et tant de poésie défile ainsi de la poubelle à l'égout tu rêves des caravelles au bord d'hypothèses et de poètes qui démontrent sur le fil des falaises la caresse de la mort sur l'échiquier des solitudes l'enfant et l'exilé ivres de la douceur d'un problème 
s'endorment sur les rives marcescentes du mystère

ô mais comment résoudre

la prose d'un nouveau poème sinon par le voyage dans l'absence où les fleuves comme de longs reptiles donnent au soleil encerclés par l'encens

d'un temps encore trop froid

Je veux crier tout le mal

que fait un tel exil

chaque fois que je me penche

pour te faire un bateau en papier

tu te souviens sans doute

de l'aube du printemps

et du souffle encore glacé

qui faisait frémir les frères exilés

du jardin des plantes

et de ta mère forgeant pour l'avenir

tes indispensables photos

et toi que l'erreur des mots

n'avait pas encore contaminé

tu regardais ces hauts oiseaux

rayer de leurs pattes captives

le miroir trompeur du bassin

tes yeux alors parlaient

dis-moi cet ailleurs

cet ailleurs que tu portes en toi

est-il plus beau que l'antichambre du sommeil

(«L'Antichambre du Sommeil », Sur le damier des tombes, 19-20)

Dans le poème « Retour », le poète se pose la question typique de tout émigrant, question existentielle, toujours obsédante et qui demeure sans réponse. Le retour tant souhaité serait-il un échec, le bonheur impossible d'un romantique attardé, ou la peur de l'autre, de devenir autre, d'être autre ? Le poète dans sa quête fait ainsi face au suicide métaphorique, à la folie causée par un départ sans explication et qu'il exprime, dans le poème suivant, par des questions rhétoriques :

Quel fut le but du départ de ces villages ensoleillés? 
Il n'y a pas de justice pour la mouche

capturée par l'araignée dans sa toile.

Jusqu'à quelle latitude

un homme devrait-il monter

pour laisser insectes

et villes derrière lui ?

Je connais ce temps

comme l'espace maudit des cauchemars ;

cela passera près de nous

te laissant retourner

vers la nuit parfaite.

Il y a un genre d'âme

que même la mémoire de hauts bâtiments

ne peut détruire. («Retour », Conseils aux suicidés, 13).

Absence de bonheur, impossibilité de l'atteindre : d'un côté le poète est le fils perdu de son pays; de l'autre, il est le fils égaré dans la nouvelle société canadienne. L'écriture devient pour lui le seul moyen pour se libérer de ce «temps » qu'il connaît et de «l'espace maudit des cauchemars » du passé et du présent. Le retour n'est donc plus pensable dans la nouvelle réalité où il vit et, encore moins, dans le souvenir d'un passé impossible à retrouver, mais plutôt en allant vers « la nuit parfaite », belle métaphore évoquant l'éternité, et cette nuit ne sera plus celle de la mort éternelle, mais plutôt un retour à la perfection divine.

Le discours d'Alexandre se définit souvent par des tournures oxymoriques. L'«Espérance » laissée au fond de la boîte de Pandore figure le voyage vers le havre de paix et, en même temps, signifie une sorte de regressus ad uterum qui, dans ses diverses acceptions, évoque la descente aux enfers (dans l'œuvre poétique d'Amprimoz, ce serait le suicide ou la descente dans les méandres de son labyrinthe intérieur) ou la montée vers les cieux (ce serait la quête inspirée par le modèle dantesque). Il faut donc réitérer ce principe clé de la poésie d'Amprimoz car, si le poète doit détruire ses utopies, celles de l'univers laissé derrière et celles de l'univers trouvé - en l'occurrence l'Amérique du nord -, le modèle qu'il suit ne le mènera certes pas à la mort mais plutôt à la construction d'un monde autre : le modèle est chrétien et dantesque, et le poète, s'inspirant des maîtres qui furent pour lui Virgile et Dante, devra vivre, frôlant les lisières de la folie, son enfer et son purgatoire symboliques, avant d'accéder à la lumière d'autres soleils. Rappelons ce mot de la Divine comédie, au moment où le poète italien aborde cet univers dont la description représente un véritable défi à la parole humaine : 
«Soudain le jour sembla s'être ajouté / au jour, comme si le tout-puissant / avait orné le ciel d'un deuxième soleil » (Paradis, I 345). Dans nos inoubliables entretiens, Alexandre se plaisait à citer par cœur le long poème de Dante, mais trop souvent, peut-être troublé par une sorte de pessimisme courageux, il s'entretenait sur le premier chant de L'Enfer. Le poète franco-italocanadien n'avait pas encore trouvé son aman :

Au milieu du chemin de notre vie je me retrouvai par une sylve obscure, car la droite voie m'était cachée.

Ahi dire ce qu'elle était est chose dure cette sylve atroce et âpre et forte qui dans le cœur rénove la frayeur!

Elle est si amère que mort l'est à peine plus ; Mais pour parler du bien que j'y trouvai, Je dirai des autres choses que j'y aperçus. (Inferno, « Chant $1 », 3$, ma traduction) ${ }^{1}$.

Le soleil n'est certes pas absent de la poésie d'Amprimoz. Il suffit ici de mentionner que tout un recueil (Champ solaire, 1978) est dédié à cet astre. Dans le poème «V », il l'implore, il l'invite à laisser « les enfants croire que la neige a un / cœur rouge », et à rentrer avec lui

\section{$[\ldots]$ dans cette forêt où leurs mots}

sont stalactites de silence tandis que leurs histoires s'en

vont glacer le lit du Nil

père ailé de l'antique sagesse j'écris en m'incli-

nant sous ta barque dorée

nous sommes les arbres timides qui frisson-

nent dans une pluie de doutes » (24).

Entre la forêt dantesque et le doute méthodique d'un Pascal, il tourne les yeux vers ce soleil spectateur des misères de ce monde, sorte de Deus absconditus qui se moque de la folie des hommes et de leurs passions inutiles :

J'ai rêvé de Marius et il m'a affirmé que le vieil

Homère fut le plus grand menteur quand Ulysse retourna toutes les âmes

d'Ithaca avaient oublié son nom quant au chien il était bien mort ( $« \mathrm{VI} », 32)$.

Mais laissons derrière l'univers des cauchemars dantesques ou homériques et rentrons de nouveau dans le labyrinthe des pensées et du langage amprimoziens. Aux lisières de la folie, c'est ainsi qu'il s'exprime dans le poème «La lame de la folie » : 
Les roses tirèrent leurs langues, les pierres commencèrent à faire l'œil et les chiens secouèrent les diamants de leurs yeux.

Comme la tête d'un saint

le soleil rebondit

trois fois sur la terre (Conseils aux suicidés, 14).

L'on reconnaît, dans l'imaginaire poétique bâti par la folie, des images apocalyptiques et particulièrement angoissantes. Aussi la dernière image du texte évoque-t-elle la tête de saint Paul décapité à Rome comparée à l'image du soleil éteint, de la lumière conquise par les ténèbres - une sorte de Genèse à l'envers, de l' «Espérance » anéantie au fond opaque de la célèbre boîte mythique de l'univers. Rappelons que du point de vue phénoménologique, l'image pourrait bien décrire la chose, mais le texte sous-tend aussi sa propre littérarité : cette tête coupée est la cause figurée du jaillissement des Trois fontaines à Rome. Le signe n'est pas référentiel, il est traditionnel et la légende romaine ne pourra être dévoilée et comprise que grâce à la clé symbolique de la parole poétique amprimozienne. Cependant, comme Heidegger et Derrida, le poète franco-romain fait du logos l'origine de la vérité discursive et l'illusion littéraire engendre l'illusion métaphysique. Une dialectique bloquée sous-tend ici la littérarité : la métaphorisation des «roses », des «pierres» et des «chiens », et l'antonomase «saint». Une progression symbolique peut être ainsi établie (Viselli 29) : par le truchement de l'oxymore, le poète évoque l'œil qui, du point de vue platonicien, n'est que le miroir de l'âme et donc de la vie éternelle, certes une référence astucieuse aux premiers mots de la Genèse et à l'Évangile selon Saint Jean. La tête du saint décapité engendre la mort physique, mais contrairement aux textes sacrés, elle évoque aussi bien la chute du soleil, donc le triomphe des ténèbres. Il y a, cependant, une discordance dans le texte amprimozien : l'article indéfini «un ». Le poète place le discours à un niveau qui dépasse la légende et élargit le champ d'interprétation à tout être singulier et extraordinaire, indéterminé - et le poète en est un, car, comme «un saint », lui aussi lance un défi sonore aux institutions, aux lois linguistiques et rhétoriques en vigueur, à la morale traditionnelle comme à la politique : cet «albatros » transplanté au Canada connaît le monde et ses secrets, sait chanter la beauté et ses nuances, et percer les voiles du statu quo poétique. Il ne faut donc pas faire une lecture trop hâtive du texte amprimozien. En fait, de la langue, l'on va progressivement vers la lumière, vers la transparence du paradis dantesque, donc vers l'univocité de la parole vraie. Les pierres perdent de leur opacité (autre thème biblique très significatif) pour 
atteindre l'hyperbole même de la pureté symbolisée par les «diamants ». Par conséquent, la langue est améliorée, sémiotiquement parlant, pour devenir lumière, soleil, le signe le plus sonore de la vie. Cependant, le mouvement vers la vie engendrée par la parole-lumière ne se fait guère sans obstacles. Nous assistons à la chute soudaine de la vie lorsque le poète la compare à la tête d'un saint décapité. Le soleil comme la tête coupée est ici condamné à rebondir trois fois sur la terre pour s'arrêter enfin sans mouvement, sans vie. Ce mouvement marque le retour inévitable à l'opacité de la terre, donc de la pierre stérile, à l'entropie et à la désolation de l'univers pré-adamique. Mais, comme nous l'avons déjà mentionné plus tôt, cette interprétation ne saurait être complète sans la dimension mythique, voire symbolique de cette mort devenue légendaire. La mort, le sang versé donne en effet naissance à un autre élément vital : l'eau. L'interprétant de la vie en tant que signe textuel, c'est l'eau qui se présente dans ce poème sous forme d'hypogramme. Rappelons donc que pour Michel Riffaterre, le texte poétique est structuré afin de répéter, sous diverses variantes, un même invariant : cet invariant est le noyau sémantique du texte, auquel Riffaterre donnera le nom d'hypogramme. Cet hypogramme, qui engendre et oriente l'écriture poétique, en constitue la matrice, l'isotopie qui permet de trouver une des clés de l'œuvre du poète. La clé du discours amprimozien semble bien être dans ce poème d'ordre métalinguistique. Mythe bien ancré dans les croyances romaines, saint Paul fut décapité sous Néron, car, en tant que citoyen romain, il ne pouvait être crucifié. Sa tête, en se détachant du corps, rebondit trois fois sur la terre, opérant ainsi le miracle des Trois fontaines, qui jaillirent du roc. Le poète, à l'exemple de saint Paul, opère grâce à l'écriture son miracle à lui. Par le truchement de la poésie (l'eau-parole régénératrice du poème), il célèbre l'heureux mariage de l'eau et de la lumière et vainc la mort. La parole poétique a donc un rôle logocentrique bien évident: elle cache et elle révèle. L'eau, la lumière et la parole poétique représentent, chez Amprimoz, le dogme démystifié, l'énigme dévoilée de la folie de la croix et de la Trinité, un mouvement certain vers le salut. Le poème «La lame de la folie » constitue une continuation logique de Changements de tons, où le poète annonce, justement, son désir de sortir $\mathrm{du}$ «labyrinthe » linguistique et psychologique de son état d'émigrant, voire d'exilé, étranger à cette société nord-américaine. Le poète, soulignons-le, vient de livrer sa guerre au silence, ou plutôt au mutisme, donc à la mort. Cependant, s'il entrevoit le soupirail de la lumière rédemptrice, il erre encore dans un «ailleurs » souhaité, son «labyrinthe » canadien. L'équilibre atteint est précaire et il risque d'échouer. Le centre tant convoité demeure toujours l'ailleurs, 
géographie vague et impressionniste, seulement présente dans l'absence, ce qui fait toute la force évocatrice de Conseils aux suicidés. Ainsi toute une procession d'images se juxtaposent-elles : l'ailleurs romain, l'ailleurs parisien, l'ailleurs du Midi de la France, celui du Canada. Les deux poèmes suivants sont hautement significatifs à cet égard :

je ferme les yeux contre la neige pour vous revoir palmiers grands frères écorchés sous la griffe des murailles

palmiers des cloîtres romains berceurs de prières je ferme les yeux («Tentative de prière », 24).

quand les étoiles pénètrent ma mémoire ton pas caresse encore l'allée du jardin il suffirait d'être ailleurs il suffirait de voir une abeille se poser sur une feuille de figuier

mais comment revenir dans ce pays le vent décide où tombent les nids («Toujours l'ailleurs », 25).

Malgré le ton amer et nostalgique, la profonde tristesse et même l'angoisse qui hantent et qui fécondent l'œuvre poétique d'Amprimoz, celui-ci lance, à sa manière, un défi au «silence éternel de ces espaces muets » qui avait tant effrayé Pascal (Pensées, 201-206 ; 528). «Conseils aux suicidés ", long poème qui conclut le recueil du même titre, est l'épitomé de toute une recherche et fraye la voie à Bouquet de signes, recueil qui, à notre humble avis, couronne, de la manière la plus sonore, le périple poétique et la quête philosophique d'Alexandre, l'aboutissement logique de son écriture et de son drame personnel. "Conseils aux suicidés » souligne chez lui l'obsession et le refus de la mort : tout ce que l'être fera, tout ce que le poète inventera, trouvera, chantera, ce sera " pour ne pas mourir », refrain qui scandera les étapes de sa descente aux enfers, une sorte de marche funèbre avant de remonter vers les étoiles ou les soleils (au pluriel) comme le veut souvent l'artiste. Le poète célèbre aussi symboliquement parlant la folie qui l'habite, une antiphrase bien filée pour évoquer la sagesse véritable, celle véhiculée 
surtout par un message profondément christique : le poète demeure le seul être à avoir le courage de surmonter les obstacles du langage et la «croix » des normes, d'inventer ses «théorèmes », son « algèbre », bref sa rhétorique. En voici des exemples :

\section{azur}

polyphonie débridée

terme sur lequel je reviendrai

à la tamise ténébreuse

mise en seine sénile

pour ce tendre tibre

ouvrir l'huître de tes paniers

pour ne pas mourir

quand dans vos vieilles coques

vient ce cygne qui saigne

ni rouge ni bleu

mais terre de sienne

adieu hypogramme charnu

je pars de biais

pour ne pas mourir

caresser les manuscrits de mortimer

fouetter au sang les mers mortes

pour faire flotter le sourire

des défuntes anaphores

pour battre le fer

au logocentre

de la polaire plaine

pour ne pas mourir

dans le sable des rêves

bâtir l'orchestre

les châteaux de neige

planter le drapeau du jour

et fourrer les séquences

événementielles

pour ne pas mourir

et devoir partir

en style sublime

sans maniérisme

sans tautologies

laisser roucouler les cons

et boire au jardin de dentelle

le sang du fils de cythère

dans un silence seul 
pour ne pas mourir

fauves crépuscules

accoudés au balcon

je cracherai sur l'écume

de vos soleils

pour ne pas mourir («Conseils aux suicidés », Conseils aux suicidés, 45-58).

Ces quelques exemples évoquent malgré tout un univers optimiste, profondément marqué par une vision christique du monde. Changements de tons (publiés en 1981) représente déjà, avant Conseils aux suicidés (1983), une étape obligée entre le monde des ténèbres et celui des lumières. Ce passage se place bien entendu sous le signe de la folie, celle du Christ par exemple, car «La folie est une vie qui vaut la peine d'être / hurlé », dit encore Amprimoz. (Changements, 71). Mais c'est dans Bouquet de signes que s'opère une sorte de révolte et une rédemption, car, le doute qui l'a hanté toute sa vie, se heurtera enfin contre les racines mêmes du doute, ce que le poète appelle au début du recueil «un infâme bouquet de signes » (Bouquet, 13). Cependant, un simple bouquet, métonymie de l'ostensoir - et donc du soleil, fera renaitre le sourire chez le poète et, radieux au milieu de ses fleurs », il fermera enfin les portes « aux sortilèges » du passé (Bouquet, 90) :

tu vois

c'était ici l'école de danse

regarde dans la cour

la fontaine ébréchée

la nuit me touche

de plus près ce soir

la lumière même

semble écarteler les ombres

et les oreilles me font mal

avec des voix d'enfants torturées

tu vois

c'était ici le seuil de la douleur

ma vie est presque sourde

l'écho du baiser de la morte

se fait aigu

et je me dis

que j'aimerais refaire le chemin 
menant du manuscrit

à l'arbre (« On ne remonte pas la danse », 34).

Le seuil de la douleur devra donc être franchi. Il aimerait « Refaire le chemin » pour sortir d'une impasse symbolique, mais le verbe demeure cependant au conditionnel : comment trouver le fil d'Ariane aux lisières de la vie ? Avec peine, le poète trouvera le courage d'avancer : le cri de douleur se transformera ainsi en prière. Le drame du poète, qui ne baisse jamais bannière, touche à son paroxysme dans le poème «L'autre maison » qui conclut le recueil Bouquet de signes et d'où nous avons tiré le titre de cette lecture-hommage à notre ami disparu. Le poète s'est enfin accordé l'aman, c'est-à-dire, il s'est octroyé la vie sauve, le salut et cela va «droit au cœur » : le poète assagi par l'âge et l'expérience se souvient de toutes les batailles essuyées, de tous les dangers, de cette «force pestilentielle » qui lui a tant de fois barré la route :

ô vous polypes ténébreux aux innombrables antennes inventeurs de labyrinthes m'empêcherez-vous encore longtemps d'aller droit au cœur

ô vous quotidiennes éponges amies des pythons galeux vous sombres dragons de la fatigue et vous force pestilentielle combien de fois m'avez-vous barré

la route

mais voilà

qu'aujourd'hui vous perdez votre temps

car le souvenir

n'est qu'une maison

que l'on porte dans sa voix. (90)

C'est cette «maison» et de cette «voix » que nous avons souhaité chanter dans ce texte pour rendre hommage à l'ami disparu mais qui, toujours présent, ne cesse de vivre dans son œuvre, dans notre souvenir, dans la nouvelle maison ici au Canada qu'il nous a appris à apprécier, hommage qui se veut forcément personnel, plus spirituel que philosophique, entre les murs de ce pays qui l'ont tant de fois vu enseigner, écrire, rire, vivre. Merci Alessandro mon ami. 


\section{Ouvrages cités}

Amprimoz, Alexandre L. Chant solaire, suivi de Vers ce logocentre. Sherbrooke: Naaman, 1978.

---. Changements de tons. Saint-Boniface : Plaines, 1981.

---. Conseils aux suicidés. Paris : Saint-Germain-des-Prés, 1983.

---. Sur le damier des tombes. Saint-Boniface : Blé, 1983.

---. Bouquets de signes. Ottawa : Prise de Parole, 1986.

Alighieri, Dante. La Divina Commedia. Ill. Gustavo Doré. Dir. Eugenio Camerini. Milan : Sonzogno, 1958.

---. La divine comédie. Tr. Jacqueline Risset. Paris : GF Flammarion, 2010.

Chevalier, Jean et Alain Cheerbrant. Dictionnaire des mythes et symboles, mythes, rêves, couleurs, nombres. Paris : Robert Laffont / Jupiter, 1982.

Hamilton, Edith. La mythologie. Alleur : Marabout, 1997.

La Bible œcuménique. Paris : Alliance biblique universelle - le CERF, 1977.

Riffaterre, Michel. La production du texte. Paris : Seuil, 1979.

---. Sémiotique de la poésie. Paris : Seuil, 1983.

Pascal, Blaise. Pensées. Euvres complètes. Dir. Lafuma. Paris : Seuil, 1963.

Viselli, Sante A. «La littérature italo-canadienne d'expression française : la voix du silence ». Le français dans tous ses états. CRDP de Montpellier, 16 (1991) : 24-33.

\section{NOTE}

${ }^{1}$ Nel mezzo del cammin di nostras vita Mi ritrovai per una selva oscura, Chè la diritta via era smarrita.

Ahi quanto a dir qual era è cosa dura Questa selva selvaggia ed aspra e forte, Che nel pensier rinnova la paura!

Tanto è amara, che poco è più morte :

Ma per trattar del ben ch'i' vi trovai, Diro' dell'altre cose, ch'io v'ho scorte. (Divina commedia, Inferno, Canto I ; 3). 